\title{
Transfer equation for the strain rate tensor and description of an incompressible dispersed mixture (incompressible fluid) by a system of equations of dynamic type
}

\author{
Askar Yuldashov ${ }^{1 *}$, Otabek Abdisamatov ${ }^{2}$, Behzod Abdullaev ${ }^{1}$, and Salima Dustova ${ }^{1}$ \\ ${ }^{1}$ Bukhara Branch Tashkent Institute of Irrigation and Agricultural Mechanization Engineers, Bukhara, \\ Uzbekistan \\ ${ }^{2}$ Tashkent Institute of Irrigation and Agricultural Mechanization Engineers, Tashkent, Uzbekistan
}

\begin{abstract}
It is known that the application of the vector operation rot to the equations of hydrodynamics leads to the Helmholtz-Friedman equation for a vortex. A dispersed mixture, tensor transformations are used, in a certain sense generalizing the vector operation rot, which gives more than one, a couple of equations. One of them describes the transfer of vorticity is the well-known Helmholtz-Friedman equation. The second equation was obtained for the first time, and it describes the transfer of the strain rate tensor. Any tensor decomposes into symmetric and antisymmetric parts. By definition, the symmetric part of the tensor $U$ is the strain rate tensor. The antisymmetric part of $U$ is a tensor whose components are related in a known manner to the pseudovector angular velocity.
\end{abstract}

\section{Introduction}

The objects of research are stationary and non-stationary flows of two-phase media under various conditions. The subject of research is the regularities of changes in the hydrodynamic parameters of dispersed mixtures in watercourses and their nodes.

Hydraulic structures worldwide are subject to various types of vibration damage caused by water hammers in water supply and discharge pipelines of systems. Finding a technique for reducing water hammer caused by cavitation and flow pulsation is one of the urgent problems. Therefore, the development of a safety methodology and the development of numerical forecasting methods in hydraulic structures and in water discharge systems of industrial complexes have a special character in operation. In this direction, in highly developed countries such as the USA, France, Italy, Brazil, South Korea, Russia, and other countries, intensive work is being carried out to create projects with a minimum appearance of a water hammer in water discharge and water supply pipelines. Therefore, the preservation of hydraulic structures, engineering communications from water hammers, and deformation of pipelines and their safe operation is one of today's main and difficult tasks and attracts special attention.

\footnotetext{
*Corresponding author: askar021954@mail.ru
} 
Particular attention in the world is paid to the scientific basis of methods for reliable forecasting of water hammer in water outlet hydraulic structures and utilities, which affects pulsation and the need to develop an effective technology. The development of effective technology is based on the creation of pressure control in pipelines compared to the pressures of para-fluid flows, which is why the conditions of cavitation. In the developed countries of the world, in the design and construction of high-pressure hydroelectric facilities, special attention is paid to the safety of these structures. The safety of structures is carried out by eliminating high speeds in the places of local resistances of the outlet pipes. At these special points, cavitation and flow pulsation are affected, which leads to the deformation of the pipes of the outlet structures. The development of methods for assessing the reliability of the use of structures and technology improvement is of great importance in operation.

\section{Methods}

The article uses mathematical modeling of flows of multiphase continua, mathematical physics, computational and full-scale experiments. Taking into account the interaction of dispersed mixtures with variable and constant concentrations, mass transfer, and interactions of the phases of the mixture in the development of approximate analytical methods for solving problems, and their implementation, allow us to reveal the patterns of movement in pipes, canals, and reservoirs.

It is known that the application of the vector operation rot to the equations of hydrodynamics leads to the Helmholtz-Friedman equation for a vortex. In this paper, tensor transformations are applied to the equations of a dispersed mixture, in a certain sense generalizing the vector operation rot, which gives more than one, a couple of equations. One of them describes the transfer of vorticity - this is the famous Helmholtz-Friedman equation. The second equation was obtained for the first time, and it describes the transfer of the strain rate tensor.

Thanks to the equation for the strain rate tensor, a dispersed incompressible liquid can be described by a closed system of a dynamic type, containing only ordinary time derivatives of the phase coordinates of a liquid particle. The phase coordinates of such a particle are understood as the components of its position vectors $r=\left\{x_{i} \mid i=1,2,3\right\}$, speed $u=\left\{u_{i} \mid i=1,2,3\right\}$, angular rate of baptism $\operatorname{rot} \frac{u}{2} \equiv \vec{\omega}=\left\{\omega_{i} \mid i=1,2,3\right\}$, and the strain rate tensor $\stackrel{\bullet}{s}=\left\{s_{i j} \mid i, j=1,2,3\right\}$. This description of a liquid is essentially Liouville. It provides a convenient basis, for example, for extending the statistical particle method to turbulence problems. Many scientists have worked on this topic [1-20]. Perhaps such a description will be useful for the kinetic theory of turbulence.

T1. The equations for the conservation of momentum of a dispersed barotropic liquid in an external field with potential $\mathrm{P}$ are written in the following form [2-4] (summation is assumed over the breaking indices).

$$
\frac{\partial u_{i}}{\partial t}+u_{k} \frac{\partial u_{i}}{\partial x_{k}}+\frac{\partial \phi}{\partial x_{1}}=0, \phi \equiv \Pi+P, i=1,2,3
$$

Here the pressure function 


$$
P(p)=\int \frac{d p}{\rho(p)}
$$

Differentiating (1) with respect to, we obtain the equations for the derivatives $\frac{\partial u_{i}}{\partial x_{i}} \equiv U_{i j}$, which are the components of the tensor $U \equiv \frac{\partial u}{\partial r}$,

$$
\frac{\partial U_{i j}}{\partial t}+u_{k} \frac{\partial U_{i j}}{\partial x_{k}}+U_{i k} U_{k j}+\frac{\partial^{2} \phi}{\partial x_{j} \partial x_{i}}=0
$$

Suppose we use the concept of the total derivative with respect to time $t$ and the notation $a: b$ for the dyadic multiplication of vectors $a$ and $b$. In that case, the last tensor equation can be rewritten in the following non-index form:

$$
\frac{\partial U}{\partial t}+U^{2}+(\vec{\nabla}: \vec{\nabla}) \phi=0
$$

Any tensor, including $U$ and the left-hand side of (2), decomposes into symmetric and antisymmetric parts. The symmetric part of the tensor $U$ by definition

$$
\operatorname{rot} \vec{U}=\left|\begin{array}{ccc}
\vec{i} & \overrightarrow{\mathrm{j}} & \overrightarrow{\mathrm{k}} \\
\frac{\partial}{\partial x}, & \frac{\partial}{\partial y}, & \frac{\partial}{\partial z} \\
\mathrm{u} & \vartheta & \mathrm{w}
\end{array}\right|
$$

strain rates

$$
\dot{S}=\frac{1}{2}\left(U+U^{*}\right), U_{i j}^{*} \equiv U_{i j}
$$

The antisymmetric part of $U$ is the tensor

$$
\dot{\Phi}=\frac{1}{2}\left(U-U^{*}\right)
$$

whose components are related in a known manner to the pseudovector $\dot{\vec{\omega}} \equiv \frac{(\text { rotu })}{2}$, how exactly

$$
\dot{\phi}_{11}=\dot{\phi}_{22}=\phi_{33} \dot{ }=0, \dot{\phi}_{12}=-\dot{\phi}_{21}=\omega_{3}, \phi_{13}=-\dot{\phi}_{31}=\omega_{2}, \dot{\phi}_{23}=-\phi_{32}=-\omega_{1} .
$$

It is not hard to see that 


$$
U^{2}=\left(\dot{\bullet} \dot{S}^{2}+\dot{\phi}^{2}\right)+\left(\begin{array}{c}
\ddot{\bullet} \dot{0} \\
\phi
\end{array}\right)
$$

Where the first expression in brackets is the symmetric part of the tensor $U^{2}$, and the second is the antisymmetric part $U^{2}$, equating to zero the symmetric part of the tensor on the left side of (2), taking into account (5), we obtain the equation for the strain rate tensor $\dot{S}$.

$$
\frac{\bullet}{d t}+\dot{s} \dot{s}^{2}+\phi^{2}+(\nabla: \nabla) \phi=0,
$$

Equating the antisymmetric part (2) to zero will give

$$
\frac{d \dot{\phi}}{d t}+\dot{\phi} \dot{S}+\dot{\bullet} \phi=0
$$

Equation (7) is the Helmholtz-Friedman equations in tensor notation. Indeed, since it is antisymmetric, it is equivalent to three independent equations of the following pseudovector equation:

$$
\frac{d \vec{\omega}}{d t}-\dot{S} \vec{\omega}+\vec{\omega} S_{p} \dot{S}=0
$$

Obviously $S_{p} \dot{S} \equiv S_{i i}=\dot{d i v u}$. and, moreover, the identity $\dot{S} \vec{\omega}=(\vec{\omega} . \nabla) u$. [2]. Thus, equation (8) is reduced to the form $\frac{d \vec{\omega}}{d t}=(\vec{\omega} \cdot \nabla) u+\vec{\omega} \cdot d i v u=0$. Coinciding with the usual vector form of the Helmholtz-Friedmann uranium. $S_{p} S=0$, In the case of an incompressible fluid and (8) implies the Helmholtz equation in the following well-known form [2]:

$$
\frac{d \vec{\omega}}{d t}=\dot{S} \vec{\omega}
$$

In conclusion of this paragraph, we note that differentiating the equation of motion (1) and separating the antisymmetric part from the obtained tensor equation (2) is equivalent to the direct application of the vector operation curl to (1), as is usually done to obtain the vorticity equation. Transformations in tensor form allow us to extract from the equation of motion (1) the equation for the tensor that is paired to the Helmholtz-Friedman equation $\dot{S}$ 
divu $=S_{p} \dot{S}=0$, and $P=\frac{p}{\rho}$, for prosperity, we assume the absence of external fields, $P=0$, then, applying the operation $S_{p}$, to $(1,6)$, we obtain the equation for the pressure function $P S_{P} \dot{S}^{2}+S_{P} \dot{\phi}^{2}+\nabla^{2} P=0$.

Obviously, $S_{P} \dot{S}^{2}=\dot{S}_{i j} \cdot \dot{S}_{j i} \equiv|S|^{2}$. the second quadratic tensor invariant $\dot{S}$ a similar tensor invariant $\dot{\phi}$ is equal $S_{P} \dot{\phi}^{2}=-2 \omega^{2}$. In this way, for $P$ we obtain the following Poisson equations:

$$
\nabla^{2} P=-e
$$

Where $e \equiv|\dot{S}|^{2}-2 \omega^{2}$ - invariant with respect to transformations of the coordinate system.

Consider a flow in an unbounded simply connected region and such that an absolutely integrable function, $\int|e(t, r)| d r<+\infty$; this takes place, for example, if outside a sphere of a certain radius $R_{0}$ fluid flow is fairly well approximated by homogeneous or shear flow. Then solution (10) can be represented by the volume potential

$$
P(t, r)=\frac{1}{4 \pi} \int \frac{e\left(t, r^{\prime}\right)}{\left|r-r^{\prime}\right|} d r^{\prime}=\frac{1}{4 \pi} \int \frac{e^{\prime}}{|R|} d R, 2 \partial e R \equiv r-r^{\prime},
$$

Expression (11) allows calculating the tensor $(\nabla: \nabla) \varphi=(\nabla: \nabla) P$, included in uranium (1.6) for $\dot{S}$ Just use the formula

$$
(\nabla: \nabla) \frac{1}{|R|}=\frac{3}{|R|^{S}}\left(R: R-\frac{1}{3} R^{2} E\right)-\frac{4 \pi}{3} \delta(R) \cdot E
$$

Wherein $E$ is the unit tensor, and the term with $\delta$ - function takes into account the nature of the singularity at the point $R=0$ second derivatives of functions $\frac{1}{|R|}$. Formula (12) can be obtained by methods of the theory of generalized functions [4] and is a generalization of the well-known identity

$$
\nabla^{2} \frac{1}{|R|}=-4 \pi \delta(R)
$$


its application allows you to make an operation $(\nabla: \nabla)$ under the integral sign (11). Calculating in this way $(\nabla: \nabla) P$, substituting the result into $(1,6)$ and using the representation $\dot{\phi}$ as $\dot{\phi}=\vec{\omega} \cdot \vec{\omega}=\omega^{2} E$, we finally get that

$$
\begin{aligned}
& \frac{d \dot{S}}{d t}+\left(\dot{S^{2}}-\frac{1}{3}|\dot{S}|^{2} E\right)+\left(\vec{\omega}: \vec{\omega}-\frac{1}{3} \omega^{2} E\right)=F \\
& F=-\frac{3}{4 \pi} \int \frac{e^{\prime}}{|R|^{5}}\left(R: R-\frac{1}{3} R^{2} E\right) d R
\end{aligned}
$$

Equation (13), together with the Helmholtz equation (9), determines the evolution of the "internal" phase coordinates $\vec{\omega}$ and $\dot{S}$ liquid particle and, consequently, the tensor invariant $\mathrm{c}$, which can be interpreted as a scalar characteristic of the proper motion of a liquid particle (deformation and rotation). Transfer of a liquid dispersed particle as a whole in physical space $\left\{x_{i} \mid i=1,2,3\right\}$ is determined by uranium (1), in which

$$
-\nabla \varphi=-\nabla P=\frac{1}{4 \pi} \int \frac{e^{\prime} R}{|R|^{3}} d R \equiv f .
$$

And so, to the Euler equations with incompressibility conditions, there corresponds a closed dynamical system of equations of the form

$$
\begin{aligned}
& \frac{d \dot{S}}{d t}+\left(S^{2}-\frac{1}{3}|\dot{S}|^{2} E\right)+\left(\vec{\omega}: \vec{\omega}-\frac{1}{3} \omega^{2} E\right)=F . \\
& \frac{d u}{d t}=f, \frac{d \vec{\omega}}{d t}=\dot{S} \vec{\omega}
\end{aligned}
$$

In which the vector $f$ and tensor $F$ are integral transformations of the function $e(t, r)=|\dot{S}|^{2} \omega^{2}$. Incompressibility condition $d i v u=0$. contained in the equations for $\dot{S} \quad$ As you read it: $\frac{d}{d t} S_{p} \dot{S}=0$. t. e $\operatorname{divu}=S_{p} \dot{S}=0 . \quad$ always, if that's the case at the beginning of time.

The system (14) allows you to present the dispersal mixture (liquid) as particle multipliers. Power $f$ determining the movement of particles in physical space is long acting, such as coulomb forces or gravitational forces. Thus, the dispersive particles that make up the solid environment of an incompressible dispersant fluid move in a self-agreed "charge" density $e$. The possibility of such an interpretation of the movement of an incompressible liquid in the physical space was previously indicated in the work of [5] based on the analogy of Euler's equations and Vlahos's kinetic uranium for ions in a selfagreed field with potential $P$. 
The systems' own movement of the dispersal particle with fluid particles (dispersion and deformation) is pollinated by the second and third equations of the system (14). Helmholtz's equation reflects the law of preserving the moment of movement of a liquid particle (change in the shape of the body and therefore its moment of inertia leads to a corresponding change in the rate of influence of this body).

\section{Conclusions}

The equation obtained in this paper links the evolution of the "shape" of a liquid particle set by the components of the tensor $\dot{S}$ with the condition of insimaceability and the influence of the following forces. These are, first, the forces of inertia that act on the elements of the particles; Second, strength $F$, has a tensor character and describes the influence of surrounding particles on the components of the tensor $\dot{S}$ It is important that the latter is a short-acting force, falling as a $R^{-3}$ when $R \rightarrow \infty$.

Thus, the dynamic system (14) allows interpreting liquid as a system of interacting particles. This reveals the re-activation of the statistical particle method [2] to hydrodynamic problems and the simulation of the turbulent transport. It is known that in the dynamics of rarefied gases, the static method of particles turned out to be one of the most effective methods of numerical modeling of complex tasks [6]. It is possible that the dynamic system will also be useful for theoretical research of the mechanisms of hydrodynamic instability and the development of turbulence.

\section{References}

1. Bazarov D., Uralov B., Matyakubov B., Vokhidov O., Uljaev F., Akhmadi M. The effects of morphometric elements of the channel on hydraulic resistance of machine channels of pumping stations, IOP Conference Series, Materials Science and Engineering, 883 (2020)

2. Loitsyansky L. G. Mechanics of liquid and gas Textbook for universities. - 7th ed., Bustard, p.840, (2003)

3. Vladimirov V.S. Generalized functions in mathematical physics. Science (1979).

4. Kadamtsev BB, Kantarovich VM - Izv. Universities, Radifizika, 17, № 4, (1974)

5. Belocerkovsky O.M., Erofeev A.N., Yanitsky V.E. -ZhVM and MF, № 5, (1980)

6. Landau L.D., Livshits E.M., Hydrodynamics. Moscow, Nauka, (1986)

7. Matyakubov B., Begmatov I., Raimova I., Teplova G. "Factors for the efficient use of water distribution facilities" IOP Conf. Series: Materials Science and Engineering $\mathbf{8 8 3}$ pp. 012050 (2020) doi:10.1088/1757-899X/883/1/012050.

8. Matyakubov B. How efficient irrigation can ensure water supply in the Lower Amudarya basin of Uzbekistan, International Water and Irrigation, 23 (3), pp. 26-27. (2003)

9. Rahmatov M., Matyakubov B., Berdiev M., Maintainability of a self-pressurized closed irrigation network, IOP Conference Series, Materials Science and Engineering, 1030 pp. 012170, (2021) doi:10.1088/1757-899X/1030/1/012170

10. Uralov B., Xidirov S., Matyakubov B., Eshonkulov Z., Norkulov B., Gayur A., River channel deformations in the area of damless water intake, IOP Conf. Series, Materials Science and Engineering $\mathbf{8 6 9}$ pp. 072014, (2020) doi:10.1088/1757899X/869/7/072014

11. Smirnov A.F. Statistical and dynamic stability of structures, 1987 
12. Yuldashev A.A., Khudaykulov S.I., Paluanov D.T. Mathematical problems of perturbation of particles of precipitation of a dispersed mixture, Istiklol: Abstracts of the international conference, pp. 286-292, Navoi, (2008)

13. Fayzullaev D.F. Pulsating motion of two-phase media in an anular pipe in the presence of near-wall layers Hydrogasdynamics, Coll. scientific. works., p. 35, Tashkent, (1986)

14. Nigmatulin R.I. Dynamics of multiphase media, p. 464, Moscow, (1987)

15. Sharipov L.A., Imomov S.J., Majitov J.A., Pulatova F., Abdisamatov O.S. Modeling of heat exchange processes in the Metanetka bioenergy plant for individual use, IOP Conference Series, Earth and Environmental Science, 614(1), pp. 012035, (2020)

16. Shodmonova G., Islomov U., Abdisamatov O., Kholiyorov U., Khamraeva S., Numerical solution of nonlinear integro-differential equations, IOP Conference Series, Materials Science and Engineering, 896(1), pp.012117. (2020)

17. Khamidov F.R., Imomov S.J., Abdisamatov O.S., Ibragimova G.Kh., Kurbonova K.I., Optimization of agricultural lands in land equipment projects, Journal of Critical Reviews, 7(11), pp.1021-1023. (2020)

18. Begmatov I.A., Matyakubov B.Sh., Akhmatov D.E., Pulatova M.V., Analysis of saline land and determination of the level of salinity of irrigated lands with use of the geographic information system technologies Inter Carto. Inter GIS GI SUPPORT OF SUSTAINABLE DEVELOPMENT OF TERRITORIES Proceedings of the International conference, 26 pp. 309 - 316, (2020)

19. Bazarov, D.R., Vokhidov, O.F., Lutsenko, L.A., Sultanov, S. Restrictions Applied When Solving One-Dimensional Hydrodynamic Equations, Lecture Notes in Civil Engineering, 70, pp. 299-305, (2020)

20. Matyakubov B.Sh., Mamatkulov Z.J., Oymatov R.K., Komilov U.N., Eshchanova G.E Assessment of the reclamation conditions of irrigated areas by geospatial analysis and recommendations for their improvement" // InterCarto. InterGIS GI SUPPORT OF SUSTAINABLE DEVELOPMENT OF TERRITORIES Proceedings of the International conference, 26, pp. 229 - 239, (2020) 